\title{
DURFEE RECTANGLES AND PSEUDO-WRONSKIAN EQUIVALENCES FOR HERMITE POLYNOMIALS
}

\author{
DAVID GÓMEZ-ULLATE, YVES GRANDATI, AND ROBERT MILSON
}

\begin{abstract}
We study an equivalence class of iterated rational Darboux transformations applied on the harmonic oscillator, showing that many choices of state adding and state deleting transformations lead to the same transformed potential. As a by-product, we derive new identities between determinants whose entries are Hermite polynomials. These identities have a combinatorial interpretation in terms of Maya diagrams, partitions and Durfee rectangles, and serve to characterize the equivalence class of rational Darboux transformations. Since the determinants have different orders, we analyze the problem of finding the minimal order determinant in each equivalence class, or equivalently, the minimum number of Darboux transformations. The solution to this problem has an elegant graphical interpretation. The results are applied to provide alternative and more efficient representations for exceptional Hermite polynomials and rational solutions of the Painlevé IV equation.
\end{abstract}

\section{INTRODUCTION}

In this paper we introduce an infinite number of identities between determinants whose entries are Hermite polynomials. The remarkable fact is that the identities involve determinants of different size. An example of such an identity would be given by

$$
\left|\begin{array}{cccc}
H_{1} & H_{1}^{\prime} & H_{1}^{\prime \prime} & H_{1}^{\prime \prime \prime} \\
H_{2} & H_{2}^{\prime} & H_{2}^{\prime \prime} & H_{2}^{\prime \prime \prime} \\
H_{3} & H_{3}^{\prime} & H_{3}^{\prime \prime} & H_{3}^{\prime \prime \prime} \\
H_{6} & H_{6}^{\prime} & H_{6}^{\prime \prime} & H_{6}^{\prime \prime \prime}
\end{array}\right|=\frac{1}{48}\left|\begin{array}{ccc}
\tilde{H}_{1} & \tilde{H}_{1}^{\prime} & \tilde{H}_{1}^{\prime \prime} \\
\tilde{H}_{2} & \tilde{H}_{2}^{\prime} & \tilde{H}_{2}^{\prime \prime} \\
\tilde{H}_{6} & \tilde{H}_{6}^{\prime} & \tilde{H}_{6}^{\prime \prime}
\end{array}\right|=\frac{1}{7680}\left|\begin{array}{cc}
H_{2} & H_{2}^{\prime} \\
\tilde{H}_{3} & \tilde{H}_{4}
\end{array}\right|
$$

where $H_{n}(x)$ denotes the $n$-th Hermite polynomial and $\tilde{H}_{n}(x)=\mathrm{i}^{-n} H_{n}(\mathrm{i} x)$. The first two determinants are Wronskian determinants, and this type of identities had already been shown in [14], and possibly known before 42 . As we shall explain below, they are a particular case of the whole equivalence class described in this paper that involves a given partition and its conjugate partition.

The third determinant in (1) is not a Wronskian, but can be constructed in a similar fashion and thus we have coined the name pseudo-Wronskian for it. A pseudo-Wronskian involves two sequences, one of ordinary Hermite polynomials $H_{n}$ and one of conjugate Hermite polynomials $\tilde{H}_{n}$. The determinant is built by taking derivatives of the ordinary Hermite polynomials as in the usual Wronskian, but shifting upwards the degree (which is almost an integration) for the conjugate Hermite polynomials.

Wronskian determinants are a key object in the theory of linear differential equations, but they also arise naturally when iterating Darboux transformations in Schrödinger's equation, as it was shown by Crum [10. This factorization method has found many applications [11, ranging from the theory of integrable systems [17, 55], soliton theory [18,39] and bispectral problems [6, 12, 30], among others. 
Darboux transformations are also fundamentally related to exceptional orthogonal polynomials, 20,23, and it was precisely this research that motivated the results reported in this paper. The determinantal identities discussed here express an equivalence class of rational Darboux transformations that lead to the same transformed potential up to a spectral shift, and the two families of entries $\left(H_{n}\right.$ and $\left.\tilde{H}_{n}\right)$ correspond to the two families of seed functions for state-adding and statedeleting rational Darboux transformations in the harmonic oscillator.

At the Schrödinger picture, these equivalences had already been noticed by in the Laguerre and Jacobi cases by Odake in [43], and by the authors in [36. The most convenient way to visualize the equivalence is by shifting the origin in a Maya diagram, a type of diagram that was originally introduced by Sato in integrable systems theory [47]. They were applied to the context of Darboux transformations and exceptional polynomials by Takemura [53].

Wronskian determinants of Hermite polynomials appear already in the work of Karlin and Szegö 37, who provide expressions for the number of real zeros of the Wronskian of a sequence of consecutive Hermite polynomials. Those sequences for which the Wronskian determinant has no real zeros were characterized by Adler in [1], and the result for arbitrary sequences has been recently derived by García-Ferrero et al. [19], using oscillatory type arguments. The complex zeros of these Wronskians of Hermite polynomials display very intriguing symmetric patterns on the complex plane, 8, 14, 15.

Beyond their obvious interest in Sturm-Liouville theory, Wronskian determinants of classical polynomials play a role in the construction of rational solutions to nonlinear integrable equations of Painlevé type. The symmetry approach based on Bäcklund transformations of Painlevé equations developed by Noumi and his collaborators (see [41] and references therein) shows how to construct rational solutions by applying the symmetry group to a number of seed solutions. Maya diagrams and partitions are also an essential part of their description. In particular, two families of rational solutions to $\mathrm{P}_{\mathrm{IV}}$ can be constructed using generalized Hermite and Okamoto polynomials, which can both be expressed as Wronskians of specific sequences of Hermite polynomials [9]. We show how to apply our results to provide an alternative pseudo-Wronskian representation of Okamoto and generalized Hermite polynomials, which happens to be more efficient in the former case.

It is also possible to view the pseudo-Wronskian determinants introduced in this paper as an extension of Jacobi-Trudi formulas in the theory of symmetric functions, 16 . The original JacobiTrudi formulas express the Schur polynomial $s_{\lambda}$ associated to a given partition $\lambda$ as a determinant whose entries are complete homogeneous symmetric polynomials, or as a determinant whose entries are elementary symmetric polynomials associated to the conjugate partition $\lambda^{\prime}$. Jacobi-Trudi formulas were already extended to include classical orthogonal polynomials in [51] and lead to exceptional orthogonal polynomials in 29]. The pseudo-Wronskian determinants in this paper can be regarded as a generalization of Jacobi-Trudi formulas that involve not just the partition $\lambda$ or its conjugate partition $\lambda^{\prime}$, but also mixed representations described by the Durfee symbols introduced by Andrews in 3. Analogous results for the Laguerre and Jacobi classes, including appropriately defined pseudo-Wronskians, will be presented in $[28$.

The paper is organized as follows: in Section 2 we introduce the basic definitions and we review the connection between partitions and Maya diagrams. In Section 3 we show how to associate a pseudo-Wronskian determinant to each labelled Maya diagram and we prove the main theorem stating the proportionality of pseudo-Wronskian determinants for all labelled Maya diagrams in the same equivalence class. In Section 4 we address the problem of finding the minimal order pseudo-Wronskian determinant in each equivalence class. Finally, in the last two sections we apply 
our results to derive alternative and more efficient pseudo-Wronskian representations of exceptional Hermite polynomials and special polynomials related to rational solutions of Painlevé type equations.

\section{DEFinitions AND PRELIMINARIES}

Definition 2.1. We define a Maya diagram to be a set of integers $M \subset \mathbb{Z}$ that contains a finite number of positive integers, and excludes a finite number of negative integers.

Definition 2.2. It is clear that if $M$ is a Maya diagram, then for $k \in \mathbb{Z}$ so is $M+k=\{m+k: m \in$ $M\}$. We say that $M$ and $M+k$ are equivalent Maya diagrams, and we define an unlabelled Maya diagram to be the equivalence class of Maya diagrams related by such shifts.

We visualize a Maya diagram as a horizontally extended sequence of filled and empty boxes, with an origin placed between the box in position -1 and box in position 0 , and with filled boxes indicating membership in $M$. By contrast, an unlabelled Maya diagram should be visualized as a sequence of filled and unfilled boxes, without a choice of origin. In this formulation, the defining assumption of a Maya diagram is that all boxes sufficiently far to the left are filled, and that all boxes sufficiently far to the right are empty. From a physical point of view, a Maya diagram depicts the empty and filled energy levels corresponding to the spectrum of a Hamiltonian with a pure point spectrum. At the filled energy levels the Hamitonian would have a bound state, leading to a state-deleting rational Darboux transformations while at the empty levels there would be a quasi-rational formal eigenfunction, leading to a rational state-adding transformation ${ }^{1}$

Definition 2.3. We say that a Maya diagram $M \subset \mathbb{Z}$ is in standard form if $0 \notin M$ and $k \in M$ for every $k<0$.

Visually, a standard diagram has a gap just to the right of the origin, and no gaps to the left of the origin.

Proposition 2.1. Let $M \subset \mathbb{Z}$ be a Maya diagram. Then there exists a unique $k \in \mathbb{Z}$ such that $M-k$ is in standard form.

Proof. The desired shift is given by $k=\min \mathbb{Z} \backslash M$.

Definition 2.4. A partition $\lambda$ is a non-increasing sequence of integers $\lambda_{1} \geq \lambda_{2} \geq \cdots$ such that $\lambda_{i}=0$ for sufficiently large $i$. Let $\ell$ be the largest index such that $\lambda_{\ell}>0$. We call $\ell$ the length of the partition. We call $|\lambda|=\lambda_{1}+\cdots+\lambda_{\ell}$ the size of the partition.

Let $M \subset \mathbb{Z}$ be a Maya diagram and let $m_{1}>m_{2}>\cdots$ be its elements ordered in decreasing order. Consider the partition defined by

$$
\lambda_{i}=\#\left\{m \notin M: m<m_{i}\right\}, \quad i=1,2 \ldots
$$

Proposition 2.2. The correspondence $M \mapsto \lambda$ given by (2) defines a bijection between the set of unlabelled Maya diagrams and the set of partitions.

\footnotetext{
${ }^{1}$ For simplicity we restrict in this paper to the case of the harmonic potential, for which only state-adding and state-deleting rational Darboux transformations exist. We postpone the analysis of hamiltonians with rational isospectral Darboux transformations to a forthcoming publication.
} 


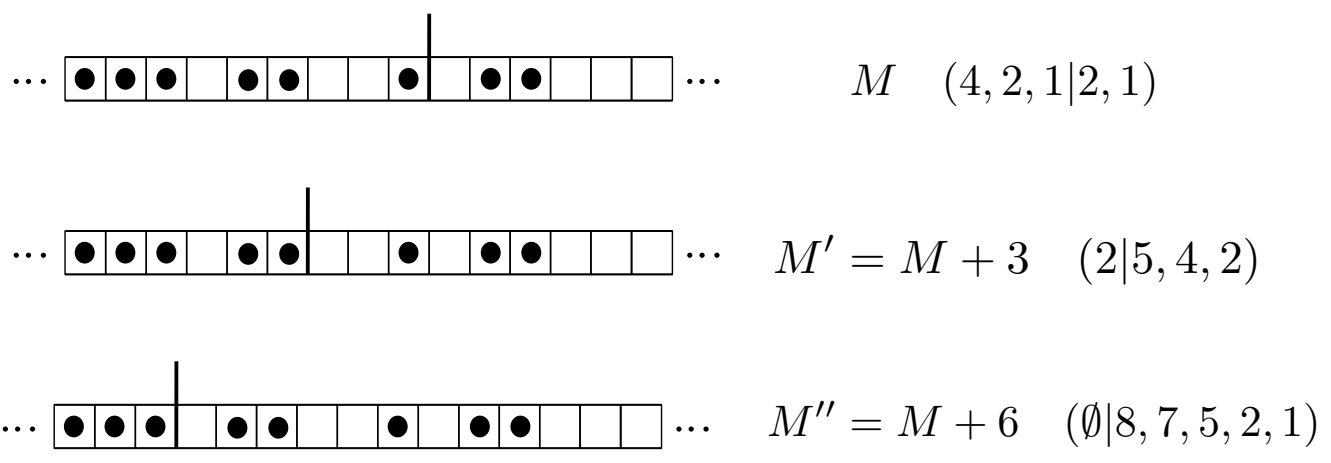

FiguRE 1. Three equivalent Maya diagrams corresponding to the partition $\lambda=$ $(4,4,3,1,1)$, together with their Frobenius representation. The third diagram is in standard form.

Proof. Observe that $M+k$ defines the same partition as $M$. By Proposition 2.1 every equivalence class of Maya diagrams contains a unique representative in standard form. Thus, it suffices to establish a 1-1 correspondence between partitions and standard diagrams. The desired correspondence is given by the relation

$$
m_{i}-\lambda_{i}=\ell-i, \quad i=1,2, \ldots
$$

If the above relation holds, then $\lambda$ is a partition of length $\ell$ if and only if $M$ is a standard diagram with $\ell$ positive elements.

It is convenient to represent a partition by means of a Ferrer's diagram, a finite collection of points arranged in left-justified rows, with the row lengths in non-increasing order.

Definition 2.5. Let $\prec$ denote the box partial order on $\mathbb{N} \times \mathbb{N}$. Formally, $\left(i_{1}, j_{1}\right) \prec\left(i_{2}, j_{2}\right)$ if and only if $i_{1} \leq i_{2}$ and $j_{1} \leq j_{2}$. We now define a Ferrer's diagram to be a finite subset of $\mathbb{N} \times \mathbb{N} 2$ which is down-closed with respect to the box order.

Formally, the correspondence between Ferrer's diagrams and partitions is as follows. Given a Ferrer's diagram $F \subset \mathbb{N} \times \mathbb{N}$, let

$$
\lambda_{j}=\#\{i \in \mathbb{N}:(i, j) \in F\}, \quad j=1,2, \ldots
$$

i.e., $\lambda_{j}$ is the number of points in row $j$. Conversely, if $\lambda$ is a partition, then the corresponding Ferrer's diagram is given by

eq:Ffromlambda

$$
F=\left\{(i, j) \in \mathbb{N} \times \mathbb{N}: i \leq \lambda_{j}\right\} .
$$

Definition 2.6. For a partition $\lambda=\left(\lambda_{1}, \ldots, \lambda_{\ell}\right)$ of length $\ell$, set

$$
\lambda_{j}^{\prime}=\#\left\{i \in \mathbb{N}: \lambda_{i} \geq j\right\}, \quad j=1,2, \ldots
$$

The resulting sequence $\lambda^{\prime}$ is called the conjugate partition of $\lambda$. The following is well known.

\footnotetext{
${ }^{2}$ Throughout the paper we will use the following notation: $\mathbb{N}=\{1,2, \ldots\}$ and $\mathbb{N}_{0}=\{0,1,2, \ldots\}$.
} 
DURFEE RECTANGLES AND PSEUDO-WRONSKIAN EQUIVALENCES FOR HERMITE POLYNOMIALS

Proposition 2.3. Let $\lambda, \lambda^{\prime}$ be as above, and let $F, F^{\prime}$ be the corresponding Ferrer's diagrams. Then $F^{\prime}$ is the transpose of $F$, meaning that

$$
F^{\prime}=\{(j, i) \in \mathbb{N} \times \mathbb{N}:(i, j) \in F .\} .
$$

There is another way to represent Maya diagrams, one that makes the relation to Ferrer's diagrams more explicit, and which will be useful when we consider the minimal order problem.

Definition 2.7. We define a bent Maya diagram to be a doubly infinite sequence $B=\left\{\left(i_{n}, j_{n}\right) \in\right.$ $\left.\mathbb{N}_{0} \times \mathbb{N}_{0}: n \in \mathbb{Z}\right\}$ such that

$$
\left(i_{n+1}, j_{n+1}\right)-\left(i_{n}, j_{n}\right) \in\{(1,0),(0,-1)\}, \quad n \in \mathbb{Z}
$$

and such that $i_{n} j_{n}=0$ for all but finitely many $n$.

Note that since the displacement $\left(i_{n}, j_{n}\right) \mapsto\left(i_{n+1}, j_{n+1}\right)$ is either down or to the right, the above definition implies that $i_{n}=0$ for all $n$ sufficiently small and that $j_{n}=0$ for all $n$ sufficiently large.

Proposition 2.4. For a Maya diagram $M \subset \mathbb{Z}$ set

$$
i_{n}=\#\{m \notin M: m<n\}, \quad j_{n}=\#\{m \in M: m \geq n\}, \quad n \in \mathbb{Z} .
$$

Then, the doubly infinite sequence $B=\left\{\left(i_{n}, j_{n}\right)\right\}_{n \in \mathbb{Z}}$ is a bent Maya diagram.

Proof. By assumption, there exists an $N>0$ such that $n \notin M$ for all $n \geq N$ and such that $n \in M$ for all $n \leq-N$. Hence, $j_{n}=0$ for all $n \geq N$ and $i_{n}=0$ for all $n \leq-N$. If $n \in M$, then $\left(i_{n+1}, j_{n+1}\right)=\left(i_{n}, j_{n}-1\right)$. If $n \notin M$, then $\left(i_{n+1}, j_{n+1}\right)=\left(i_{n}+1, j_{n+1}\right)$. Therefore, in both cases the defining condition of a bent diagram is satisfied.

Informally, a bent Maya diagram is a 2-dimensional representation of a Maya diagram, with a filled box at position $n$ corresponding to a unit downward displacement $(0,-1)$ and an empty box corresponding to a unit rightward displacement $(1,0)$, as depicted in Figure 2 A translation $M^{\prime}=M-k$ corresponds to an index shift in the bent diagram:

$$
\left(i_{n}^{\prime}, j_{n}^{\prime}\right)=\left(i_{n+k}, j_{n+k}\right) \text {. }
$$

There is a connection between bent diagrams and Ferrer's diagrams.

Definition 2.8. Let $F \subset \mathbb{N} \times \mathbb{N}$ be a Ferrer's diagram. We define the rim of $F$ to be subset

$$
F^{\prime}=\{(i, j) \in F:(i+1, j+1) \notin F\} .
$$

Proposition 2.5. Let $M \subset \mathbb{Z}$ be a Maya diagram, $B$ the corresponding bent diagram defined by (6), $\lambda$ the corresponding partition defined by (2), and $F$ the corresponding Ferrer's diagram defined by (4). Then, $F^{\prime}=B \cap(\mathbb{N} \times \mathbb{N})$.

Thus, the rim is that finite subset of the bent diagram whose points have non-zero coordinates.

Proof. We first show that the non-zero part of $B$ lies in $F^{\prime}$. Suppose that $i_{n}, j_{n}>0$. Let $m_{1}>$ $m_{2}>\cdots$ be the elements of $M$ in decreasing order. By (6), $m_{j_{n}+1}<n \leq m_{j_{n}}$. Since

$$
\left\{m \notin M: m<m_{j_{n}+1}\right\} \subset\{m \notin M: m<n\} \subset\left\{m \notin M: m<m_{j_{n}}\right\}
$$

it follows that $\lambda_{j_{n}+1} \leq i_{n} \leq \lambda_{j_{n}}$. Therefore by (4), $\left(i_{n}, j_{n}\right) \in F$ but $\left(i_{n}+1, j_{n}+1\right) \notin F$.

We now prove the converse. Arguing by contradiction, suppose that there is an $(i, j) \in F^{\prime}$ which does not belong to $B$. In $F^{\prime} \backslash B$ choose the points with $j$ as small as possible, and of those choose the point that has $i$ as large as possible. By assumption, $i \leq \lambda_{j}$. We consider two cases. 
Case 1: assume that $i<\lambda_{j}$. Then $i+1 \leq \lambda_{j}$, which means that $(i+1, j) \in F$. Since $(i+1, j+1) \notin F$, the same is true for $(i+2, j+1)$. Hence, $(i+1, j) \in F^{\prime}$ also. Because of the assumed maximality of $i$, we must have $(i+1, j) \in B$; i.e. $(i+1, j)=\left(i_{n}, j_{n}\right)$ for some $n \in \mathbb{Z}$. By the definition of a bent diagram, $\left(i_{n-1}, j_{n-1}\right)$ is either $\left(i_{n}, j_{n}+1\right)=(i+1, j+1)$ or $\left(i_{n}-1, j_{n}\right)=(i, j)$. The second possibility is excluded because we have assumed that $(i, j) \notin B$. By the first part of the proof, $\left(i_{n-1}, j_{n-1}\right) \in F^{\prime}$. This means that $(i+1, j+1) \in F$, which contradicts the assumption that $(i, j) \in F^{\prime}$.

Case 2: assume that $i=\lambda_{j}$. If $j>1$, then $i \leq \lambda_{j-1}$ which means that $(i, j-1) \in F$. By assumption, $(i+1, j) \notin F$. Hence $(i, j-1) \in F^{\prime}$ and hence $(i, j-1) \in B$ by the minimality of $j$. We now repeat the above argument to conclude that $(i, j) \in B$ also - a contradiction. Hence $j=1$ and $i=\lambda_{1}$. Set $n=\max M$. By (2), $i_{n}=\lambda_{1}$ and $j_{n}=1$. This contradicts the assumption that $(i, j) \notin B$.

Definition 2.9. For a given Maya diagram $M \subset \mathbb{Z}$, define

$$
M_{+}=\{m \in M: m \geq 0\}, \quad M_{-}=\{-m-1: m<0, m \notin M\} .
$$

In other words, $M_{+}$gives positions of the filled boxes to the right of the origin, and $M_{-}$the positions of the holes to the left of the origin. The numbers in $M_{+}$and $M_{-}$indicate distance to the origin, with 0 indicating a position adjacent to the origin. Since $M_{+}$and $M_{-}$fully define $M$, the defining assumptions of a Maya diagram are equivalent to the condition that $M_{+}$and $M_{-}$should be finite subsets of $\mathbb{N}_{0}$.

Definition 2.10. Let $M \subset \mathbb{Z}$ be a Maya diagram. Let $\left\{s_{1}, \ldots, s_{p}\right\}, p=i_{0}$, be the elements of $M_{-}$and $\left\{t_{1}, \ldots, t_{q}\right\}, q=j_{0}$, the elements of $M_{+}$, arranged in decreasing order. The double list $\left(s_{1}, \ldots, s_{p} \mid t_{1}, \ldots, t_{q}\right)$ is called the Frobenius symbol of $M$ [4].

The classical Frobenius symbol $3,5,44]$ corresponds to the case where $i_{0}=j_{0}$; i.e. the case where $M_{-}$and $M_{+}$have the same cardinality. Such a choice of origin can be visualized as the unique intersection of the rim and the main diagonal in $\mathbb{N} \times \mathbb{N}$.

Let us also note the following connection between the Frobenius symbol and bent diagrams.

Proposition 2.6. Let $M \subset \mathbb{Z}$ be a Maya diagram and $B=\left\{\left(i_{n}, j_{n}\right)\right\}_{n \in \mathbb{Z}}$ the corresponding bent diagram. Then, $i_{n}$ and $j_{n}$ are the cardinalities of $(M-n)_{-}$and $(M-n)_{+}$, respectively.

Proof. By (6), $i_{0}$ is the cardinality of $M_{-}$and $j_{0}$ the cardinality of $M_{+}$. The general relation follows by (7).

\section{Hermite pseudo-Wronskians}

In this section we will associate to each labelled Maya diagram a certain determinant whose entries are Hermite polynomials. We then prove that determinants associated to equivalent Maya diagrams are proportional to each other. This is our main result in this Section.

For $n \geq 0$, let

eq:Hndef

$$
H_{n}(x)=(-1)^{n} e^{x^{2}} D_{x}^{n} e^{-x^{2}}, \quad D_{x}=\frac{d}{d x}
$$

denote the degree $n$ Hermite polynomial, and

$$
\tilde{H}_{n}(x)=\mathrm{i}^{-n} H_{n}(\mathrm{i} x)
$$




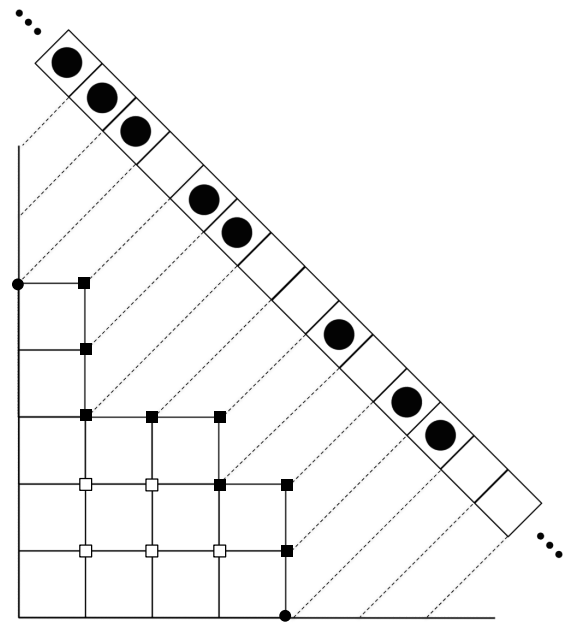

FiguRE 2. Correspondence between an unlabelled Maya diagram and a partition $\lambda=(4,4,3,1,1)$. The squares are points that belong to the Ferrer diagram $F$, while black squares belong to the rim of $F$.

the conjugate Hermite polynomial. Recall that $y=H_{n}$ is a solution of the Hermite differential equation

eq:hermiteDE

$\operatorname{def}: \mathrm{HpW}$

eq:pWdef 1

$$
y^{\prime \prime}-2 x y^{\prime}+2 n y=0
$$

for $n \geq 0$, and that $y=e^{x^{2}} \tilde{H}_{-n-1}$ is a solution of 10 for $n<0$.

Definition 3.1. Let $M \subset \mathbb{Z}$ be a Maya diagram. Let $\left\{s_{1}, \ldots, s_{p}\right\}$ be the elements of $M_{-}$and $\left\{t_{1}, \ldots, t_{q}\right\}$ the elements of $M_{+}$, both arranged in descending order. We define the Hermite pseudoWronskian associated to $M$ to be

$$
H_{M}=e^{-p x^{2}} \operatorname{Wr}\left[e^{x^{2}} \tilde{H}_{s_{1}}, \ldots, e^{x^{2}} \tilde{H}_{s_{p}}, H_{t_{q}}, \ldots H_{t_{1}}\right],
$$

where Wr denotes the Wronskian determinant of the indicated functions.

The polynomial nature of $H_{M}$ becomes evident once we represent it using a slightly different determinant.

Proposition 3.1. A Hermite pseudo-Wronskian admits the following alternative determinantal representation

$$
H_{M}=\left|\begin{array}{cccc}
\tilde{H}_{s_{1}} & \tilde{H}_{s_{1}+1} & \ldots & \tilde{H}_{s_{1}+p+q-1} \\
\vdots & \vdots & \ddots & \vdots \\
\tilde{H}_{s_{p}} & \tilde{H}_{s_{p}+1} & \ldots & \tilde{H}_{s_{p}+p+q-1} \\
H_{t_{q}} & D_{x} H_{t_{q}} & \ldots & D_{x}^{p+q-1} H_{t_{q}} \\
\vdots & \vdots & \ddots & \vdots \\
H_{t_{1}} & D_{x} H_{t_{1}} & \ldots & D_{x}^{p+q-1} H_{t_{1}}
\end{array}\right|
$$


Proof. The desired conclusion follows by the fundamental identities

eq:hermids

eq:Wrhomog

$$
\begin{aligned}
& D_{x} H_{n}(x)=2 n H_{n-1}(x), \quad n \geq 0, \\
& D_{x} \tilde{H}_{n}(x)=2 n \tilde{H}_{n-1}(x), \quad n \geq 0, \\
& 2 x H_{n}(x)=H_{n+1}(x)+2 n H_{n-1}(x), \\
& 2 x \tilde{H}_{n}(x)=\tilde{H}_{n+1}(x)-2 n \tilde{H}_{n-1}(x), \\
& D_{x}\left(e^{x^{2}} \tilde{H}_{n}(x)\right)=e^{x^{2}} \tilde{H}_{n+1}(x), \\
& D_{x}\left(e^{-x^{2}} h_{n}(x)\right)=-e^{-x^{2}} h_{n+1}(x) .
\end{aligned}
$$

and the Wronskian identity

$$
\mathrm{Wr}\left[g f_{1}, \ldots, g f_{s}\right]=g^{s} \operatorname{Wr}\left[f_{1}, \ldots, f_{s}\right]
$$

We refer to $H_{M}$ as a pseudo-Wronskian because it is constructed by means of a modified Wronskian operator that replaces $D_{x}$ with an indicial shift for the rows with the conjugate Hermites. More specifically, if $j \in M_{+}$, then the $(j, k)$ entry is $D_{x}^{(k-1)} H_{j}$, as in the ordinary Wronskian. If $i \in M_{-}$, then the $(i, k)$ entry is the conjugate Hermite polynomial $\tilde{H}_{i+k-1}$.

Example 3.1. Consider the first Maya diagram in Figure1. The Frobenius symbol is $(5,2,1 \mid 2,1)$. The Hermite pseudo-Wronskian $H_{M}$ associated to $M$ is given by

$$
H_{M}=\mathrm{e}^{-3 x^{2}} \operatorname{Wr}\left[\mathrm{e}^{x^{2}} \tilde{H}_{5}, \mathrm{e}^{x^{2}} \tilde{H}_{2}, \mathrm{e}^{x^{2}} \tilde{H}_{1}, H_{1}, H_{2}\right]=\left|\begin{array}{ccccc}
\tilde{H}_{5} & \tilde{H}_{6} & \tilde{H}_{7} & \tilde{H}_{8} & \tilde{H}_{9} \\
\tilde{H}_{2} & \tilde{H}_{3} & \tilde{H}_{4} & \tilde{H}_{5} & \tilde{H}_{6} \\
\tilde{H}_{1} & \tilde{H}_{2} & \tilde{H}_{3} & \tilde{H}_{4} & \tilde{H}_{5} \\
H_{1} & H_{1}^{\prime} & H_{1}^{\prime \prime} & H_{1}^{\prime \prime \prime} & H_{1}^{(4)} \\
H_{2} & H_{2}^{\prime} & H_{2}^{\prime \prime} & H_{2}^{\prime \prime \prime} & H_{2}^{(4)}
\end{array}\right|
$$

The main result of this section is the following class of fundamental determinantal identities enjoyed by these polynomials

thm: detequiv

Theorem 3.1. Let $M$ and $M^{\prime}=M-k, k>0$ be two equivalent Maya diagrams. Set

$$
\begin{array}{rlrl}
E_{k} & =\{m \in M: 0 \leq m<k\} & G_{k} & =\{m \notin M: 0 \leq m<k\} \\
\epsilon_{i} & =(-1)^{\#\{m \notin M: m<i\}} \prod_{\substack{m \in M \\
m>i}}(2 m-2 i) & \gamma_{i}=(-1)^{\#\{m \in M: m>i\}} \prod_{\substack{m \notin M \\
m<i}}(2 m-2 i)
\end{array}
$$

Then,

eq: detequiv

$$
\left(\prod_{i \in G_{k}} \gamma_{i}\right) H_{M^{\prime}}=\left(\prod_{i \in E_{k}} \epsilon_{i}\right) H_{M}
$$

Proof. Once the following two Lemmas are established, it suffices to verify that the factors shown in (16) and (18) below are equal to the above-defined $\gamma_{i}$ and $\epsilon_{i}$ symbols, respectively.

Throughout, $\left\{s_{1}, \ldots, s_{p}\right\}$ and $\left\{t_{1}, \ldots, t_{q}\right\}$ are, respectively, the elements of $M_{-}$and $M_{+}$arranged in descending order. 
lem:shift1 Lemma 3.1. Suppose that $M^{\prime}=M-1$ and that $0 \in M$. Then,

eq: shift1

$$
H_{M}=(-1)^{p} 2^{q-1}\left(\prod_{b=1}^{q-1} t_{b}\right) H_{M^{\prime}} .
$$

Proof. By assumption, $t_{q}=0$ and

$$
M_{-}^{\prime}=\left\{s_{1}+1, \ldots, s_{p}+1\right\}, \quad M_{+}^{\prime}=\left\{t_{1}-1, \ldots, t_{q-1}-1\right\} .
$$

The identity

eq:Wr1 (17)

$$
\mathrm{Wr}\left[1, f_{1}, \ldots, f_{s}\right]=\mathrm{Wr}\left[D f_{1}, \ldots, D f_{s}\right]
$$

together with 13 implies that

$$
\begin{aligned}
H_{M} & =e^{-p x^{2}} \operatorname{Wr}\left[e^{x^{2}} \tilde{H}_{s_{1}}, \ldots, e^{x^{2}} \tilde{H}_{s_{p}}, 1, H_{t_{q-1}}, \ldots, H_{t_{q}}\right] \\
& =(-1)^{p} e^{-p x^{2}} \operatorname{Wr}\left[D\left(e^{x^{2}} \tilde{H}_{s_{1}}\right), \ldots, D\left(e^{x^{2}} \tilde{H}_{s_{p}}\right), D H_{t_{q-1}}, \ldots, D H_{t_{1}}\right] \\
& =(-1)^{p} 2^{q}\left(\prod_{b=1}^{q-1} t_{b}\right) e^{-p x^{2}} \operatorname{Wr}\left[e^{x^{2}} \tilde{H}_{s_{1}+1}, \ldots, e^{x^{2}} \tilde{H}_{s_{p}+1}, H_{t_{q-1}-1}, \ldots, H_{t_{1}-1}\right]
\end{aligned}
$$

lem:shift2 Lemma 3.2. Suppose that $M^{\prime}=M+1$ and that $-1 \notin M$. Then,

eq: shift2

$$
H_{M}=(-1)^{p+q-1} 2^{p-1}\left(\prod_{a=1}^{p-1} s_{a}\right) H_{M^{\prime}}
$$

Proof. By assumption, $s_{p}=0$ and

$$
M_{-}^{\prime}=\left\{s_{1}-1, \ldots, s_{p-1}-1\right\}, \quad M_{+}^{\prime}=\left\{t_{1}+1, \ldots, t_{q}+1\right\} .
$$

The identity 14 together with 17) implies

$$
\begin{aligned}
H_{M} & =e^{-p x^{2}} \operatorname{Wr}\left[e^{x^{2}} \tilde{H}_{s_{1}}, \ldots, e^{x^{2}} \tilde{H}_{s_{p-1}}, e^{x^{2}}, h_{t_{q}}, \ldots H_{t_{1}}\right] \\
& =e^{q x^{2}} \operatorname{Wr}\left[\tilde{H}_{s_{1}}, \ldots, \tilde{H}_{s_{p-1}}, 1, e^{-x^{2}} h_{t_{q}}, \ldots, e^{-x^{2}} H_{t_{1}}\right] \\
& =(-1)^{p-1} e^{q x^{2}} \operatorname{Wr}\left[D \tilde{H}_{s_{1}}, \ldots, D \tilde{H}_{s_{p-1}}, D e^{-x^{2}} h_{t_{q}}, \ldots, D e^{-x^{2}} H_{t_{1}}\right] \\
& =(-1)^{p+q-1} 2^{p-1}\left(\prod_{a=1}^{p-1} s_{a}\right) e^{q x^{2}} \operatorname{Wr}\left[\tilde{H}_{s_{1}-1}, \ldots, \tilde{H}_{s_{p-1}-1}, e^{-x^{2}} h_{t_{q}+1}, \ldots, e^{-x^{2}} H_{t_{1}+1}\right] \\
& =(-1)^{p+q-1} 2^{p-1}\left(\prod_{a=1}^{p-1} s_{a}\right) e^{-(p-1) x^{2}} \operatorname{Wr}\left[e^{x^{2}} \tilde{H}_{s_{1}-1}, \ldots, e^{x^{2}} \tilde{H}_{s_{p-1}-1}, H_{t_{q}+1}, \ldots, H_{t_{1}+1}\right]
\end{aligned}
$$

Proposition 2.1 immediately gives the following.

Corollary 3.1. Every Hermite pseudo-Wronskian is a scalar multiple of a Hermite Wronskian

$$
\mathrm{Wr}\left[H_{m_{\ell}}, \ldots, H_{m_{1}}\right]
$$

for some unique choice of positive integers $m_{1}>\cdots>m_{\ell}>0$. 
Example 3.2. Consider the three equivalent Maya diagrams in Figure 1. We have

$$
\begin{aligned}
& M^{\prime}=M+3: \quad M_{-}^{\prime}=\{2\} \quad M_{+}^{\prime}=\{2,4,5\} \\
& M^{\prime \prime}=M+6: \quad M_{-}^{\prime \prime}=\emptyset \quad M_{+}^{\prime \prime}=\{1,2,5,7,8\}
\end{aligned}
$$

Hence,

$$
\begin{aligned}
H_{M^{\prime}} & =\left|\begin{array}{llll}
\tilde{H}_{2} & \tilde{H}_{3} & \tilde{H}_{4} & \tilde{H}_{5} \\
H_{2} & H_{2}^{\prime} & H_{2}^{\prime \prime} & H_{2}^{\prime \prime \prime} \\
H_{4} & H_{4}^{\prime} & H_{4}^{\prime \prime} & H_{4}^{\prime \prime \prime} \\
H_{5} & H_{5}^{\prime} & H_{5}^{\prime \prime} & H_{5}^{\prime \prime \prime}
\end{array}\right|, \\
H_{M^{\prime \prime}} & =\operatorname{Wr}\left[H_{1}, H_{2}, H_{5}, H_{7}, H_{8}\right]
\end{aligned}
$$

By 15 ,

$$
-483840 H_{M^{\prime \prime}}=-1935360 H_{M^{\prime}}=H_{M} .
$$

Before continuing, we mention that a "pure" pseudo-Wronskian corresponding to a Maya diagram without any positive elements can also be expressed as a Wronskian determinant of conjugate Hermite polynomials.

Proposition 3.2. Let $M \subset \mathbb{Z}$ be a Maya diagram consisting entirely of negative integers, that is $M_{+}=\emptyset$. Let $\left\{s_{1}, \ldots, s_{p}\right\}$ be the elements of $M_{-}$arranged in descending order. Then,

$$
H_{M}=\mathrm{Wr}\left[\tilde{H}_{s_{1}}, \ldots, \tilde{H}_{s_{p}}\right]
$$

Proof. Recall that

$$
H_{M}=e^{-p x^{2}} \operatorname{Wr}\left[e^{x^{2}} \tilde{H}_{s_{1}}, \ldots, e^{x^{2}} \tilde{H}_{s_{p}}\right]
$$

The desired identity now follows using (14).

As a special case of Theorem 3.1 we obtain the following identity; see [42] 14 and the references therein.

Corollary 3.2. Let $\lambda$ be a partition of length $\ell, \lambda^{\prime}$ the conjugate partitions of length $\ell^{\prime}=\lambda_{1}$. Let $m_{1}>\ldots>m_{\ell}$ the positive integers defined by $(3)$ and $m_{1}^{\prime}>\ldots>m_{\ell^{\prime}}^{\prime}$ the analogous integers for $\lambda^{\prime}$. Then,

$$
2^{\ell^{\prime}} V\left(m_{1}^{\prime}, \ldots, m_{\ell^{\prime}}^{\prime}\right) \operatorname{Wr}\left[H_{m_{1}}, \ldots, H_{m_{\ell}}\right]=2^{\ell} V\left(m_{1}, \ldots, m_{\ell}\right) \operatorname{Wr}\left[\tilde{H}_{m_{1}^{\prime}}, \ldots, \tilde{H}_{m_{\ell^{\prime}}}\right]
$$

where

$$
V\left(a_{1}, \ldots, a_{k}\right)=\prod_{1 \leq i<j<k}\left(a_{j}-a_{i}\right)
$$

is the usual Vandermonde determinant.

\section{THE MINIMAL ORDER PROBLEM}

It is quite remarkable to have an infinite family of identities among determinants of different order. In this section we pose and solve the following question: given an unlabelled Maya diagram, which of the corresponding equivalent pseudo-Wronskian determinants has the smallest order? This question will have some applications in the following sections to derive simpler, alternative representations of certain class of special functions. The precise formulation of the question requires the following. 
Definition 4.1. We define $|M|$, the girth of a Maya diagram $M$, to be the length of its Frobenius symbol, that is the sum of the cardinalities of $M_{-}$and $M_{+}$. Since the girth of $M$ is just the order of the corresponding pseudo-Wronskian determinant $H_{M}$, our aim is to determine

$$
\min \{|M-k|: k \in \mathbb{Z}\},
$$

a quantity that we will call the minimal girth of $M$. A $k \in \mathbb{Z}$ such that $|M-k|$ is minimal will be called a minimal girth origin for $M$.

As we now show, it suffices to check for minimal girth at a finite number of $k$-values.

Proposition 4.1. If $k \in \mathbb{Z}$ is a minimal girth origin for a Maya diagram $M$, then necessarily $k-1 \in M$ and $k \notin M$.

More plainly, the minimal girth origins of a Maya diagram must occur at locations where a full box is succeeded by an empty box.

Proof. Let $\left(s_{1}, \ldots, s_{p} \mid t_{1}, \ldots, t_{q}\right)$ be the Frobenius symbol of $M-k$. If $k \in M$, then $t_{q}=0$. Observe that the Frobenius symbol of $M^{\prime}=M-k-1$ is $\left(s_{1}+1, \ldots, s_{p}+1 \mid t_{1}-1, \cdots, t_{q-1}-1\right)$. If $k-1 \notin M$, then $s_{p}=0$. In this case, observe that the Frobenius symbol of $M^{\prime}=M-k+1$ is $\left(s_{1}-1, \ldots, s_{p-1}-1 \mid t_{1}+1, \ldots, t_{q}+1\right)$. In both cases, $\left|M^{\prime}\right|=|M-k|-1$. Therefore $k \notin M$ and $k-1 \in M$.

The solution of the minimal order problem is closely related to the geometry of Ferrer's diagrams.

Definition 4.2. Let $F \subset \mathbb{N} \times \mathbb{N}$ be a Ferrer's diagram corresponding to a partition $\lambda$. We call $(i, j) \in F$ an inside corner if $(i, j+1),(i+1, j) \in F$ but $(i+1, j+1) \notin F$.

Proposition 4.2. Let $B=\left\{\left(i_{n}, j_{n}\right)\right\}_{n \in \mathbb{Z}}$ be the bent diagram corresponding to a Maya diagram $M \subset \mathbb{Z}$. Then $|M-n|=i_{n}+j_{n}$.

Proof. Recall that $i_{n}$ is the cardinality of $(M-n)_{-}$while $j_{n}$ is the cardinality of $(M-n)_{+}$.

Proposition 4.3. Let $n \in Z$ be a minimal girth origin for a Maya diagram $M \subset \mathbb{Z}$ corresponding to a partition $\lambda=\left(\lambda_{1}, \ldots, \lambda_{\ell}\right)$. Then, one of the following three possibilities holds:

(a) $\left(i_{n}, j_{n}\right)$ is an inside corner of the corresponding Ferrer's diagram,

(b) $\left(i_{n}, j_{n}\right)=(0, \ell)$

(c) $\left(i_{n}, j_{n}\right)=\left(\lambda_{1}, 0\right)$.

Proof. Without loss of generality, assume that $M$ is in standard form. By Proposition 4.1, $n-1 \in$ $M$ and $n \notin M$. Hence, by (6), $\left(i_{n-1}, j_{n-1}\right)=\left(i_{n}, j_{n}-1\right)$ and $\left(i_{n+1}, j_{n+1}\right)=\left(i_{n}+1, j_{n}\right)$. If $0<n<\max M$, then $i_{n}, j_{n}>0$ and hence by Proposition 2.5. $\left(i_{n}, j_{n}\right)$ is an inside corner of $F$. The only other possibilities are $n=0$ and $n=m_{1}+1$, where $m_{1}=\max M$. In the former case, $\left(i_{0}, j_{0}\right)=(0, \ell)$. In the second case, $\left(i_{m_{1}+1}, j_{m_{1}+1}\right)=\left(\lambda_{1}, 0\right)$.

cor:MGO Corollary 4.1. Let $M \subset \mathbb{Z}$ be a Maya diagram and $\lambda$ the corresponding partition. Then, the minimal girth is given by $\min \left\{\lambda_{j+1}+j: 0 \leq j \leq \ell\right\}$.

Proof. By (4) the inside corners of the corresponding Ferrer's diagram occur at $\left(\lambda_{j+1}, j\right)$ for $j=$ $1, \ldots, \ell-1$. If $j=0$, then $\left(\lambda_{j+1}, j\right)=\left(\lambda_{1}, 0\right)$. If $j=\ell$, then $\left(\lambda_{j+1}, j\right)=(0, \ell)$. The desired conclusion now follows by Proposition 4.3 


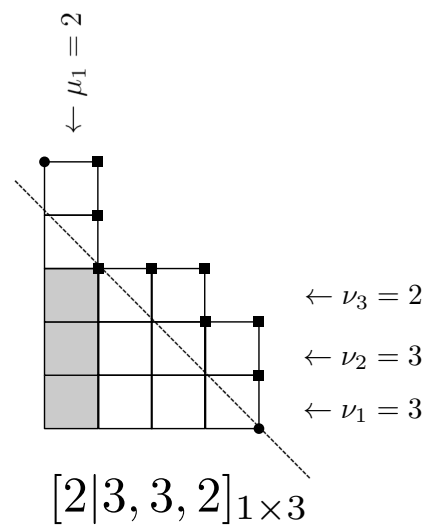

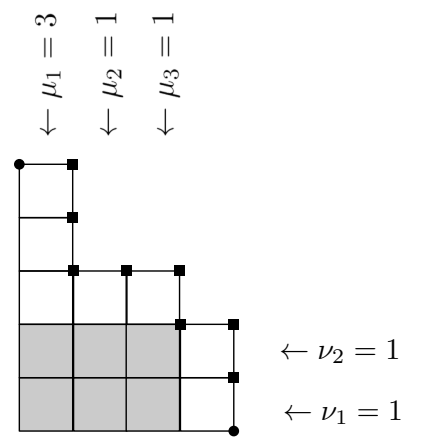

$[3,1,1 \mid 1,1]_{3 \times 2} \quad[\emptyset \mid 4,4,3,1,1]_{0 \times 5}$

FiguRE 3. Durfee rectangles and Durfee symbols for the three equivalent Maya diagrams depicted in Figure 11. Note that the shortest girth corresponds to the left diagram, which is a non-unique solution to the minimal order problem for this partition. The right diagram where the Durfee symbol coincides with the partition, corresponds to the Maya diagram in standard form.

Definition 4.3. Let $F$ be a Ferrer's diagram. We define a Durfee rectangle 3$]$ of $F$ to be a rectangle with vertices $(0,0),(i, 0),(i, j),(0, j)$ where $(i, j)$ is an inside corner of $F$. If $M$ is the corresponding Maya diagram with an origin located at an inside corner, then the corresponding Frobenius symbol $\left(s_{1}, \ldots, s_{p} \mid t_{1}, \ldots, t_{q}\right)$ satisfies $s_{p}, t_{q}>0$. We are thus able to define the partitions

$$
\begin{aligned}
\mu & =\left(s_{1}-p+1, \ldots, s_{p-1}+1, s_{p}\right) \\
\nu & =\left(t_{q}-q+1, \ldots, t_{q-1}+1, t_{q}\right)
\end{aligned}
$$

of length $p$ and $q$, respectively. We call $[\mu \mid \nu]_{p \times q}$ the Durfee symbol of $M$.

Visually, the partitions $\mu$ and $\nu$ describe the complement of the Durfee rectangle in $F$ (see Figure 4). Partition $\mu$ is the transpose of the remnant above the rectangle, and $\nu$ is the remnant to the right of the rectangle. The girth of the corresponding Maya diagram is simply the distance of the inside corner to the origin relative to the taxi-cab metric. To determine the minimal girth, the corresponding girths have to be compared to the height $\ell$ of the Ferrer's diagram, and the width $\lambda_{1}$, which can be considered as Durfee rectangles of width zero and height zero, respectively (black circle dots in Figure 4 ).

\section{Application to Exceptional Hermite polynomials}

One possible application of Theorem 3.1 and the minimal order problem is to provide a more efficient computation for exceptional Hermite polynomials. Exceptional Hermite polynomials are complete families of orthogonal polynomials that arise as eigenfunctions of a Sturm-Liouville problem on the real line, 21, 22. The degree sequence of each family does not range over all positive integers, i.e. there is a finite number of gaps or missing degrees. Let $\lambda=\left(\lambda_{1}, \ldots, \lambda_{\ell}\right)$ be a partition and $M \subset \mathbb{Z}$ the corresponding standard Maya diagram with $M_{+}=\left\{m_{1}, \ldots, m_{\ell}\right\}$ its positive elements as determined by (3). Following 25,26] we define an infinite number of polynomials in the 
following manner:

: XHermitedef

eq:XDE

eqn:hatT

$$
H_{n}^{(\lambda)}=\mathrm{Wr}\left[H_{m_{\ell}}, \ldots, H_{m_{1}}, H_{\ell-|\lambda|+n}\right], \quad n \notin M+|\lambda|-\ell
$$

By construction, $H_{n}^{(\lambda)}$ is a polynomial with

$$
\operatorname{deg} H_{n}^{(\lambda)}=\sum_{i=1}^{\ell}\left(m_{i}-i+1\right)+\ell-|\lambda|+n-\ell=n
$$

The degree sequence for the exceptional Hermite family indexed by partition $\lambda$ is $\mathbb{Z} \backslash(M+|\lambda|-\ell)$. Thus, degrees $0,1, \ldots,|\lambda|-\ell-1$ and the degrees $m_{1}+|\lambda|-\ell, \ldots, m_{\ell}+|\lambda|-\ell$ are missing, so that the polynomial sequence $\left\{H_{n}^{(\lambda)}\right\}_{n}$ is missing a total of $|\lambda|$ degrees, the codimension of the sequence.

Exceptional Hermite polynomials are a generalization the classical Hermite family because they satisfy a Hermite-like differential equation

$$
T_{\lambda}\left[H_{n}^{(\lambda)}\right]=2(N-n) H_{n}^{(\lambda)}, \quad n \notin M+|\lambda|-\ell,
$$

where

$$
T_{\lambda}[y]:=y^{\prime \prime}-2\left(x+\frac{H_{M}^{\prime}}{H_{M}}\right) y^{\prime}+\left(\frac{H_{M}^{\prime \prime}}{H_{M}}+2 x \frac{H_{M}^{\prime}}{H_{M}}\right) y .
$$

We say that $\lambda$ is an even partition if $\ell$ is even and $\lambda_{2 i-1}=\lambda_{2 i}$ for every $i$. If $\lambda$ is even, then the exceptional Hermite polynomials $H_{n}^{(\lambda)}$ satisfy the orthogonality relations

$$
\int_{\mathbb{R}} H_{n}^{(\lambda)} H_{m}^{(\lambda)} W_{\lambda}(x) d x=\delta_{m, n} \sqrt{\pi} 2^{j+\ell} j ! \prod_{i=1}^{\ell}\left(j-m_{i}\right), \quad n, m \notin M+|\lambda|-\ell
$$

where $j=n+\ell-N$, and the orthogonality weight is defined as

$$
W_{\lambda}(x)=\frac{e^{-x^{2}}}{H_{M}(x)^{2}} .
$$

Moreover, if $\lambda$ is an even partition then $\operatorname{span}\left\{H_{n}^{(\lambda)}: n \notin M+|\lambda|-\ell\right\}$ is dense in $\mathrm{L}^{2}\left(\mathbb{R}, W_{\lambda}\right)$.

Exceptional polynomials appear in a number of applications in mathematical physics, mostly as solutions to exactly solvable quantum mechanical problems describing bound states $25,45,49]$. They appear also in connection with super-integrable systems 38, 48, exact solutions to Dirac's equation [50], diffusion equations and random processes [32], finite-gap potentials [31] and point vortex models 34 .

From a mathematical point of view, the main results are concerned with the full classification of exceptional polynomials 20,23 , properties of their zeros 24, 33, 35, and recurrence relations 13, 26, 40, 46.

Here we are concerned with the most economical presentation of a given exceptional Hermite polynomial family. The definition (19) involves the computation of a Wronskian determinant of order $\ell+1$. In light of the preceding results, this order can be potentially reduced by replacing the Wronskian with an appropriate pseudo-Wronskian.

Let $M \subset \mathbb{Z}$ be a Maya diagram, $m_{1}>m_{2}>\cdots$ the elements of $M$ arranged in descending order, and $\lambda$ the corresponding partition. Set

$$
O_{r}=\left\{m_{j}+1: \lambda_{j+1}+j=r, 0 \leq j \leq \ell\right\} \quad r \geq 0 .
$$


Let $k_{r}=\max O_{r}$, or $-\infty$ if the latter is the empty set. Visually, the elements of $O_{r}$ are the labels of the inside corners that lie on the anti-diagonal $i+j=r$, with $k_{r}$ the largest such. By Corollary 4.1. if $r$ is the minimal girth, then $k_{r}$ is the largest minimal girth origin.

prop:shortest

Proposition 5.1. Let $M \subset \mathbb{Z}$ be a Maya diagram, $m \notin M$ and $M^{\prime}=M \cup\{m\}$. Let $r$ be the minimal girth of $M$. Let $r^{\prime}$ denote the minimal girth of $M^{\prime}$ and $O_{r^{\prime}}^{\prime}$ the minimal girth origins of $M^{\prime}$. Then one of the following mutually exclusive possibilities holds.

(a) If $m<k_{r}$ then $r^{\prime}=r-1$ and $O_{r^{\prime}}^{\prime}=\left\{k \in O_{r}: k>m\right\}$.

(b) If $m=k_{r}$ then $r^{\prime}=r$ and $O_{r^{\prime}}^{\prime}=\{m+1\} \cup\left\{k \in O_{r+1}: k>m\right\}$.

(c) If $k_{r}<m<k_{r+1}$, then $r^{\prime}=r$ and $O_{r^{\prime}}^{\prime}=\left\{k \in O_{r+1}: k>m\right\}$.

(d) If $m \geq \max \left\{k_{r}, k_{r+1}\right\}$ then $r^{\prime}=r+1$ and $O_{r^{\prime}}^{\prime}=O_{r} \cup\left\{k \in O_{r+2}: k>m\right\}$.

Proof. Let $B=\left\{\left(i_{k}, j_{k}\right)\right\}_{k \in \mathbb{Z}}$ and $B^{\prime}=\left\{\left(i_{k}^{\prime}, j_{k}^{\prime}\right)\right\}_{k \in \mathbb{Z}}$ be the bent diagrams for $M$ and $M^{\prime}$, respectively. The proof is based on the following key observation:

$$
\left(i_{k}^{\prime}, j_{k}^{\prime}\right)= \begin{cases}\left(i_{k}, j_{k}+1\right) & \text { if } k \leq m \\ \left(i_{k}-1, j_{k}\right) & \text { if } k>m .\end{cases}
$$

We argue each of the above cases in turn. Suppose that (a) holds. If $k \in O_{r}$ and $k>m$, then $i_{k}^{\prime}+j_{k}^{\prime}=r-1$. For all other $k$, we have $i_{k}^{\prime}+j_{k} \geq r$.

Suppose that (b) holds. Hence $\left(i_{m+1}, j_{m+1}\right)=\left(i_{m}+1, j_{m}\right)$ and hence $i_{m+1}^{\prime}+j_{m+1}^{\prime}=i_{m}+j_{m}=r$. If $k \leq m$, then $i_{k}^{\prime}+j_{k}^{\prime} \geq r+1$. If $k>m$, then $i_{k}^{\prime}+j_{k}^{\prime}=i_{k}+j_{k}-1$ is equal to $r$ if $k \in O_{r+1}$ and is $>r$, otherwise.

Suppose that (c) holds. If $k \leq m$, then $i_{k}^{\prime}+j_{k}^{\prime} \geq r+1$. If $k>m$ then $i_{k}^{\prime}+j_{k}^{\prime}=r$ if $k \in O_{r+1}$ and is $>r$ otherwise.

Suppose that (d) holds. If $k \leq m$ then $i_{k}^{\prime}+j_{k}^{\prime}=r+1$ if $k \in O_{r}$ and is $>r$ otherwise. If $k>m$ then $i_{k}^{\prime}+j_{k}^{\prime}=r+1$ if $k \in O_{r+2}$ and is $>r$ otherwise.

Let $M \subset \mathbb{Z}$ be a Maya diagram and $\lambda$ the corresponding partition. For $n \notin M+|\lambda|-\ell$ set $M_{n}^{(\lambda)}=M \cup\{n+\ell-|\lambda|\}$. Observe that $H_{n}^{(\lambda)}=(-1)^{\ell} H_{M_{n}^{(\lambda)}}$. We therefore seek the origin of minimal girth for $M_{n}^{(\lambda)}$. Let $r_{n}^{(\lambda)}=\min \left\{\left|M_{n}^{(\lambda)}-k\right|: k \in \mathbb{Z}\right\}$ be the minimal order for $H_{n}^{(\lambda)}$. We call any such $k$ that realizes this minimum an origin of minimal order.

eq: shortest1

Corollary 5.1. Let $r=r_{M}$ and $n \notin M+|\lambda|-\ell$. If $k_{r+1}>k_{r}$, then

$$
r_{n}^{(\lambda)}= \begin{cases}r-1 & \text { if } n<k_{r}+|\lambda|-\ell, \\ r & \text { if } k_{r}+|\lambda|-\ell \leq n<k_{r+1}+|\lambda|-\ell \\ r+1 & \text { if } n>k_{r+1}+|\lambda|-\ell\end{cases}
$$

An origin of minimal order for each of the above cases is, respectively, $k_{r}, k_{r+1}, k_{r}$.

If $k_{r+1}<k_{r}$, then

eq: shortest2

$$
r_{n}^{(\lambda)}= \begin{cases}r-1 & \text { if } n<k_{r}+|\lambda|-\ell, \\ r & \text { if } n=k_{r}+|\lambda|-\ell \\ r+1 & \text { if } n>k_{r}+|\lambda|-\ell\end{cases}
$$

An origin of minimal order for each of the above case is, respectively, $k_{r}, k_{r}+1, k_{r}$. 
Example 5.1. Consider the case of $\lambda=(2,2,1,1)$ The corresponding Frobenius symbol is $(\emptyset \mid 1,2,4,5)$. The minimal girth is $r=2$ with $k_{2}=6$ the unique minimal girth origin. The Frobenius symbol of $M-6$ is $(5,2 \mid \emptyset)$. Hence, pseudo-Wronskian of smallest order is

$$
H_{M-6}=\left|\begin{array}{cc}
\tilde{H}_{5} & \tilde{H}_{6} \\
\tilde{H}_{2} & \tilde{H}_{3}
\end{array}\right|
$$

with

$$
H_{M}=\mathrm{Wr}\left[H_{1}, H_{2}, H_{4}, H_{5}\right]=-2^{5} \times 24 H_{M-6} .
$$

The exceptional Hermite polynomials for this partition are given by

$$
H_{n}^{(\lambda)}=\mathrm{Wr}\left[H_{1}, H_{2}, H_{4}, H_{5}, H_{n-2}\right], \quad n \in\{2,5,8,9,10, \ldots\} .
$$

We can replace the above $5 \times 5$ determinant with a pseudo-Wronskian of smaller order. Here $k_{3}=3<6$ and hence we must apply (23). An origin of minimal order is 7 if $n=7$, and 6 otherwise. Explicitly,

$$
\begin{aligned}
H_{2}^{(\lambda)} & =2^{12} \times 120 \tilde{H}_{2} \\
H_{5}^{(\lambda)} & =2^{12} \times 72 \quad \tilde{H}_{5} \\
H_{8}^{(\lambda)} & =K_{8}\left|\begin{array}{cc}
\tilde{H}_{6} & \tilde{H}_{7} \\
\tilde{H}_{3} & \tilde{H}_{4}
\end{array}\right| \\
H_{n}^{(\lambda)} & =K_{n}\left|\begin{array}{ccc}
\tilde{H}_{5} & \tilde{H}_{6} & \tilde{H}_{7} \\
\tilde{H}_{2} & \tilde{H}_{3} & \tilde{H}_{4} \\
H_{n-8} & H_{n-8}^{\prime} & H_{n-8}^{\prime \prime}
\end{array}\right|, \quad n>8,
\end{aligned}
$$

where

$$
K_{n}=-2^{9} \times 24 \times(n-3)(n-4)(n-6)(n-7)
$$

Example 5.2. Consider the case of $\lambda=(4,4,1,1)$ The corresponding Frobenius symbol is $(\emptyset \mid$ $7,6,2,1)$. The minimal girth is $r=3$ with $k_{3}=3$ the unique minimal girth origin. The Frobenius symbol of $M-3$ is $(2 \mid 4,3)$. Hence, pseudo-Wronskian of smallest order is

$$
H_{M-3}=\left|\begin{array}{ccc}
\tilde{H}_{2} & \tilde{H}_{3} & \tilde{H}_{4} \\
H_{3} & H_{3}^{\prime} & H_{3}^{\prime \prime} \\
H_{4} & H_{4}^{\prime} & H_{4}^{\prime \prime}
\end{array}\right|
$$

with

$$
H_{M}=\mathrm{Wr}\left[H_{1}, H_{2}, H_{6}, H_{7}\right]=2^{5} \times 600 H_{M-3} .
$$

The exceptional Hermite polynomials for this partition are given by

$$
H_{n}^{(\lambda)}=\mathrm{Wr}\left[H_{1}, H_{2}, H_{6}, H_{7}, H_{n-6}\right], \quad n \in\{6,9,10,11,14,15, \ldots\} .
$$


Here $k_{4}=8>3$ and hence we must apply $(22)$. An origin of minimal order is 8 if $9 \leq n \leq 14$ and 3 otherwise. Explicitly,

$$
\begin{aligned}
& H_{6}^{(\lambda)}=2^{14} \times 9 \times 7 \times 25 \mathrm{Wr}\left[H_{3}, H_{4}\right] \\
& H_{9}^{(\lambda)}=2^{11} \times 9 \times 5\left|\begin{array}{ccc}
\tilde{H}_{2} & \tilde{H}_{3} & \tilde{H}_{4} \\
\tilde{H}_{3} & \tilde{H}_{4} & \tilde{H}_{5} \\
\tilde{H}_{7} & \tilde{H}_{8} & \tilde{H}_{9}
\end{array}\right|, \\
& H_{10}^{(\lambda)}=2^{11} \times 9 \times 5\left|\begin{array}{ccc}
\tilde{H}_{2} & \tilde{H}_{3} & \tilde{H}_{4} \\
\tilde{H}_{4} & \tilde{H}_{5} & \tilde{H}_{6} \\
\tilde{H}_{7} & \tilde{H}_{8} & \tilde{H}_{9}
\end{array}\right|, \\
& H_{11}^{(\lambda)}=-2^{11} \times 3 \times 25\left|\begin{array}{cccc}
\tilde{H}_{3} & \tilde{H}_{4} & \tilde{H}_{5} \\
\tilde{H}_{4} & \tilde{H}_{5} & \tilde{H}_{6} \\
\tilde{H}_{7} & \tilde{H}_{8} & \tilde{H}_{9}
\end{array}\right|, \\
& H_{n}^{(\lambda)}=K_{n}\left|\begin{array}{cccc}
\tilde{H}_{2} & \tilde{H}_{3} & \tilde{H}_{4} & \tilde{H}_{5} \\
H_{2} & H_{2}^{\prime} & H_{2}^{\prime \prime} & H_{2}^{\prime \prime \prime} \\
H_{4} & H_{4}^{\prime} & H_{4}^{\prime \prime} & H_{4}^{\prime \prime \prime} \\
H_{n-9} & H_{n-9}^{\prime} & H_{n-9}^{\prime \prime} & H_{n-9}^{\prime \prime \prime}
\end{array}\right|, \quad n \geq 14
\end{aligned}
$$

where

$$
K_{n}=-2^{10} \times 45 \times(n-7)(n-8)
$$

\section{Application to rational solutions of Painleve IV}

In this section we apply our minimal order results to the description of rational solutions of $\mathrm{P}_{\mathrm{IV}}$, the fourth Painlevé equation:

$$
y^{\prime \prime}=\frac{\left(y^{\prime}\right)^{2}}{2 y}+\frac{3}{2} y^{3}+4 t y^{2}+2\left(t^{2}-a\right) y+\frac{b}{y}, \quad y=y(t) .
$$

In order to connect $\mathrm{P}_{\mathrm{IV}}$ to pseudo-Wronskians we need to recall the notion of a factorization chain, and to describe the class of rational solvable extensions of the harmonic oscillator.

A factorization chain is a sequence of Schrödinger operators

$$
L_{i}=-D_{x}^{2}+U_{i}, \quad D_{x}=\frac{d}{d x}, U_{i}=U_{i}(x)
$$

that is related by Darboux transformations,

eq:Dxform

$$
\begin{aligned}
L_{i} & =\left(D_{x}+f_{i}\right)\left(-D_{x}+f_{i}\right)+\lambda_{i}, \quad f_{i}=f_{i}(x), \\
L_{i+1} & =\left(-D_{x}+f_{i}\right)\left(D_{x}+f_{i}\right)+\lambda_{i} .
\end{aligned}
$$

It follows that $f_{i}$ is the solution of the Riccati equations

$$
f_{i}^{\prime}+f_{i}^{2}=U_{i}-\lambda_{i}, \quad-f_{i}^{\prime}+f_{i}^{2}=U_{i+1}-\lambda_{i} .
$$

Equivalently,

$$
L_{i} \psi_{i}=\lambda_{i} \psi_{i}, \quad \text { where } f_{i}=\frac{\psi_{i}^{\prime}}{\psi_{i}} .
$$

It also follows that the potentials of the chain are related by

$$
U_{i+n}=U_{i}-2\left(f_{i}^{\prime}+\cdots+f_{i+n-1}^{\prime}\right) .
$$


DURFEE RECTANGLES AND PSEUDO-WRONSKIAN EQUIVALENCES FOR HERMITE POLYNOMIALS 17

Eliminating the potentials, we obtain a chain of coupled equations

$$
\left(f_{i}+f_{i+1}\right)^{\prime}+f_{i+1}^{2}-f_{i}^{2}=\alpha_{i}, \quad \alpha_{i}=\lambda_{i}-\lambda_{i+1} .
$$

We speak of an $n$-step cyclic factorization chain if $U_{n+1}=U_{1}+\Delta$ for some $n \in \mathbb{N}$ and constant

$$
\Delta=-\left(\alpha_{1}+\cdots+\alpha_{n}\right) \text {. }
$$

It is well known $[2,54]$ that $\mathrm{P}_{\mathrm{IV}}$ is equivalent to the 3-step cyclic factorization chain

eq:3chain

$$
\begin{aligned}
& \left(f_{1}+f_{2}\right)^{\prime}+f_{2}^{2}-f_{1}^{2}=\alpha_{1}, \quad f_{i}=f_{i}(z), i=1,2,3 \\
& \left(f_{2}+f_{3}\right)^{\prime}+f_{3}^{2}-f_{2}^{2}=\alpha_{2} \\
& \left(f_{3}+f_{1}\right)^{\prime}+f_{1}^{2}-f_{3}^{2}=\alpha_{3}
\end{aligned}
$$

The reduction of 28 to $\mathrm{P}_{\mathrm{IV}}$ is via the following relations

eq: $f 123 W$

$$
\begin{gathered}
f_{1}=-W-\frac{\Delta}{2} z, \quad f_{2,3}=\frac{W}{2} \pm \frac{W^{\prime}-\alpha_{2}}{2 W}, \quad \Delta=-\left(\alpha_{1}+\alpha_{2}+\alpha_{3}\right), W=W(z), \\
W(z)=2^{-\frac{1}{2}} \Delta^{\frac{1}{2}} y(t), z=2^{\frac{1}{2}} \Delta^{-\frac{1}{2}} t, \quad a=\Delta^{-1}\left(\alpha_{3}-\alpha_{1}\right), b=-2 \Delta^{-2} \alpha_{2}^{2} .
\end{gathered}
$$

Next, we recall some relevant definitions from 25. A rational extension of the harmonic oscillator is a potential of the form

$$
U(x)=x^{2}+\frac{a(x)}{b(x)},
$$

where $a(x), b(x)$ are polynomials with $\operatorname{deg} a<\operatorname{deg} b$. We say that the corresponding Schrödinger operator $L=-D^{2}+U$ is exactly solvable by polynomials if there exists functions $\mu(x), \zeta(x)$ such that for all but finitely many $k \in \mathbb{N}_{0}$ there exists a degree $n$ polynomial $y_{n}(z)$ such that

$$
\psi_{n}=\mu(x) y_{n}(\zeta(x))
$$

is a (formal) eigenfunction of $L$ - that is

$$
-\psi_{n}^{\prime \prime}+U \psi_{n}=\lambda_{n} \psi_{n}
$$

for some constant $\lambda_{n}$. The following result was proved in 25].

Theorem 6.1. Every rational extension of the harmonic oscillator that is exactly solvable by polynomials has the form

$$
U(x)=x^{2}-2 D_{x}^{2} \log \mathrm{Wr}\left[H_{m_{1}}, \ldots, H_{m_{\ell}}\right]+C,
$$

where $\left\{m_{1}, \ldots, m_{\ell}\right\}$ are positive integers and $C$ is an additive constant.

For a Maya diagram $M \subset \mathbb{Z}$, define

eq:UMdef

$$
U_{M}=x^{2}-2 D_{x}^{2} \log H_{M}+2\left|M_{+}\right|-2\left|M_{-}\right| .
$$

Proposition 6.1. Let $M \subset \mathbb{Z}$ and $M^{\prime}=M+k, k \in \mathbb{Z}$ be equivalent Maya diagrams. Then

$$
U_{M^{\prime}}=U_{M}+2 k, \quad k \in \mathbb{Z} \text {. }
$$

Proof. By Theorem 3.1.

$$
D_{x}^{2} \log H_{M}=D_{x}^{2} \log H_{M^{\prime}}
$$

To establish the value of the shift in (31), it suffices to consider the case of $k=1$. The general case then follows by induction. Now there are two subcases. If $-1 \in M$ then $M_{-}^{\prime}=M_{-}$and $M_{+}^{\prime}=M_{+}+1$. If $-1 \notin M$, then $M_{-}^{\prime}=M_{-}-1$ and $M_{+}^{\prime}=M_{+}$. Therefore, in both cases,

$$
M_{+}^{\prime}-M_{-}^{\prime}=M_{+}-M_{-}+1 \text {. }
$$


Thus, the set of rational extensions of the harmonic oscillator modulo additive constants is in bijective correspondence with the set of unlabelled Maya diagrams.

Definition 6.1. We define a Maya diagram chain to be a sequence of Maya diagrams $M_{1}, M_{2}, \ldots, M_{\ell}$ such that there exist $m_{1}, \ldots, m_{\ell} \in \mathbb{Z}$ satisfying

$$
M_{i+1}=\left\{\begin{array}{ll}
M_{i} \cup\left\{m_{i}\right\} & \text { if } m_{i} \notin M_{i}, \\
M_{i} \backslash\left\{m_{i}\right\} & \text { if } m_{i} \in M_{i},
\end{array}, \quad i=1, \ldots, \ell-1 .\right.
$$

The following are proved in [27]. Also see [8] and the references therein.

Proposition 6.2. Let $M_{i}, i=1, \ldots, \ell$ be a Maya diagram chain. Then, $L_{i}=-D^{2}+U_{M_{i}}, i=$ $1, \ldots, \ell$ is a factorization chain. Conversely, every factorization chain of rational extensions of the harmonic oscillator that are solvable by polynomials arises in precisely this fashion.

Theorem 6.2. Every rational solution of $\mathrm{P}_{\mathrm{IV}}$ corresponds to a 3-cyclic chain of rational extensions of the harmonic oscillator.

In light of the above, we wish to classify 3-cyclic chains of Maya diagrams $M_{1}, M_{2}, M_{3}, M_{4}$ such that $M_{4}=M_{1}+k$ for some $k \in \mathbb{Z}$. To accomplish this, we define the following classes of Maya diagrams:

$$
\begin{gathered}
\mathrm{GH}(m, \ell)=\mathbb{Z}_{-} \cup\{j \in \mathbb{Z}: m \leq j \leq m+\ell-1\}, \quad m, \ell \in \mathbb{N}_{0}, \\
\mathrm{O}\left(\ell_{1}, \ell_{2}\right)=\mathbb{Z}_{-} \cup\left\{3 j+1: 0 \leq j<\ell_{1}\right\} \cup\left\{3 j+2: 0 \leq j<\ell_{2}\right\}, \quad \ell_{1}, \ell_{2} \in \mathbb{N}_{0},
\end{gathered}
$$

where $\mathbb{Z}_{-}$is the set of negative integers. For example,

$$
\mathrm{GH}(2,5)_{+}=\{2,3,4,5,6\}, \quad \mathrm{O}(2,5)_{+}=\{1,2,4,5,8,11,14\} .
$$

We will call the former a Maya diagram of GH-type (Generalized Hermite), and the latter, a Maya diagram of O-type (Okamoto). Before proceeding, we note the following degeneracies of this notation:

$$
\mathrm{GH}(m, 0)=\mathrm{O}(0,0)=\mathbb{Z}_{-}, \quad \mathrm{GH}(0, \ell)=\mathbb{Z}_{-}+\ell .
$$

The following results are proved in 27].

prop:MGHU

eq: GHmaya

Proposition 6.3. Suppose that $M_{i} \subset \mathbb{Z}, i=1, \ldots, 4$ is a chain of Maya diagrams such that $M_{4}=M_{1}+k$ for some $k \in \mathbb{Z}$. Then, up to translation, $M_{1}$ is either a Maya diagram of GH-type or of O-type. In the first case,

$$
M_{1}=\mathrm{GH}(m, \ell), \quad M_{4}=\left(M_{1} \backslash m\right) \cup\{0, m+\ell\}, \quad m, \ell \in \mathbb{N}_{0} .
$$

In the second case,

$$
M_{1}=\mathrm{O}\left(\ell_{1}, \ell_{2}\right), \quad M_{4}=M_{1} \cup\left\{0,3 \ell_{1}+1,3 \ell_{2}+2\right\}, \quad \ell_{1}, \ell_{2} \in \mathbb{N}_{0} .
$$


Proposition 6.4. The rational solutions of $\mathrm{P}_{\mathrm{IV}}$ that arise from 3-cyclic chains of the harmonic oscillator fall into one of two classes. The rational solutions of GH-type take the form:

eq: GH1

eq : GH2

eq: GH3

eq:U1

eq :U2

eq:U3

$$
\begin{array}{lll}
y=D_{t}\left[\log \frac{H_{\mathrm{GH}(m, \ell)}}{H_{\mathrm{GH}(m, \ell+1)}}\right]_{x=t}, & a=-(1+m+2 \ell), & b=-2 m^{2}, \\
y=D_{t}\left[\log \frac{H_{\mathrm{GH}(m, \ell)}}{H_{\mathrm{GH}(m-1, \ell)}}\right]_{x=t}, & a=2 m+\ell-1, & b=-2 \ell^{2}, m>0, \\
y=D_{t}\left[\log \frac{H_{\mathrm{GH}(m, \ell)}}{H_{\mathrm{GH}(m+1, \ell-1)}}\right]_{x=t}-2 t, & a=\ell-m-1, & b=-2(m+\ell)^{2}, \ell>0 .
\end{array}
$$

The rational solutions of O-type take the form:

$$
\begin{array}{lll}
y=-\frac{2}{3} t+D_{t}\left[\log \frac{H_{\mathrm{O}\left(\ell_{1}, \ell_{2}\right)}}{H_{\mathrm{O}\left(\ell_{1}-1, \ell_{2}-1\right)}}\right]_{x=\frac{t}{\sqrt{3}}}, & a=\ell_{1}+\ell_{2}, & b=-\frac{2}{9}\left(1-3 \ell_{1}+3 \ell_{2}\right)^{2} \\
y=-\frac{2}{3} t+D_{t}\left[\log \frac{H_{\mathrm{O}\left(\ell_{1}, \ell_{2}\right)}}{H_{\mathrm{O}\left(\ell_{1}+1, \ell_{2}\right)}}\right]_{x=\frac{t}{\sqrt{3}}}, & a=-1-2 \ell_{1}+\ell_{2}, & b=-\frac{2}{9}\left(2+3 \ell_{2}\right)^{2} \\
y=-\frac{2}{3} t+D_{t}\left[\log \frac{H_{\mathrm{O}\left(\ell_{1}, \ell_{2}\right)}}{H_{\mathrm{O}\left(\ell_{1}, \ell_{2}+1\right)}}\right]_{x=\frac{t}{\sqrt{3}}}, & a=-2-2 \ell_{2}+\ell_{1}, & b=-\frac{2}{9}\left(1+3 \ell_{1}\right)^{2}
\end{array}
$$

Since the rational solutions of $\mathrm{P}_{\mathrm{IV}}$ are linear combinations of log-derivatives of Hermite Wronskians, it makes sense to apply the shortest chain results of Section 4 to obtain more economical descriptions of these rational solutions.

prop:GH Proposition 6.5. If $m, \ell>0$, then the minimal order of a GH-type Maya diagram $M=\mathrm{GH}(m, \ell)$ is $\min \{m, \ell\}$. If $\ell \leq m$, then the minimal order determinant is just the usual Wronskian

$$
H_{M}=\mathrm{Wr}\left[H_{m}, \ldots, H_{m+\ell-1}\right] .
$$

If $\ell>m$ then the minimal-order determinant is the pseudo-Wronskian

$$
H_{M-m-\ell}=\left|\begin{array}{ccc}
\tilde{H}_{\ell} & \ldots & \tilde{H}_{\ell+m-1} \\
\vdots & \ddots & \vdots \\
\tilde{H}_{\ell+m-1} & \ldots & \tilde{H}_{\ell+2 m-2}
\end{array}\right|
$$

Example 6.1. For the rational solutions of $\mathrm{P}_{\mathrm{IV}}$ corresponding to the generalized Hermite class, take $m=2, \ell=4$ and set

$$
\begin{aligned}
& M_{0}=\mathrm{GH}(2,4)=\mathbb{Z}_{-} \cup\{2,3,4,5\} \\
& M_{1}=\mathrm{GH}(2,5)=\mathbb{Z}_{-} \cup\{2,3,4,5,6\} \\
& M_{2}=\mathrm{GH}(1,4)=\mathbb{Z}_{-} \cup\{1,2,3,4\} \\
& M_{3}=\mathrm{GH}(3,3)=\mathbb{Z}_{-} \cup\{3,4,5\} .
\end{aligned}
$$

The corresponding Wronskians are determinants of order 4,5,4,3, respectively. The more economical description is in term of pseudo-Wronskian determinants of order $2,2,1,3$, respectively. By a 
direct calculation,

$$
\begin{aligned}
H_{M_{0}-6} & =\left|\begin{array}{ll}
\tilde{H}_{5} & \tilde{H}_{6} \\
\tilde{H}_{4} & \tilde{H}_{5}
\end{array}\right|=-32\left(16 x^{8}+64 x^{6}+120 x^{4}+45\right) \\
H_{M_{1}-7} & =\left|\begin{array}{ll}
\tilde{H}_{6} & \tilde{H}_{7} \\
\tilde{H}_{5} & \tilde{H}_{6}
\end{array}\right|=-64\left(32 x^{10}+240 x^{8}+720 x^{6}+600 x^{4}+450 x^{2}-225\right) \\
H_{M_{2}-5} & =\tilde{H}_{5}=4\left(4 x^{4}+12 x^{2}+3\right) \\
H_{M_{3}-6} & =\left|\begin{array}{lll}
\tilde{H}_{5} & \tilde{H}_{6} & \tilde{H}_{7} \\
\tilde{H}_{4} & \tilde{H}_{5} & \tilde{H}_{6} \\
\tilde{H}_{3} & \tilde{H}_{4} & \tilde{H}_{5}
\end{array}\right|=-512 x\left(16 x^{8}+72 x^{4}-135\right)
\end{aligned}
$$

Setting $x=t$, the corresponding rational solutions (36) (37) (38) are

$$
\begin{array}{lll}
y_{1}=\frac{32\left(4 t^{7}+12 t^{5}+15 t^{3}\right)}{16 t^{8}+64 t^{6}+120 t^{4}+45}-\frac{20\left(16 t^{9}+96 t^{7}+216 t^{5}+120 t^{3}+45 t\right)}{32 t^{10}+240 t^{8}+720 t^{6}+600 t^{4}+450 t^{2}-225}, & (a, b)=(-11,-8), \\
y_{2}=-\frac{32\left(4 t^{7}+12 t^{5}+15 t^{3}\right)}{16 t^{8}+64 t^{6}+120 t^{4}+45}-\frac{8\left(2 t^{3}+3 t\right)}{4 t^{4}+12 t^{2}+3}, & (a, b)=(7,-32), \\
y_{3}=-\frac{1}{t}-\frac{32\left(4 t^{7}+9 t^{3}\right)}{16 t^{8}+72 t^{4}-135}+\frac{32\left(4 t^{7}+12 t^{5}+15 t^{3}\right)}{16 t^{8}+64 t^{6}+120 t^{4}+45}-2 t, & (a, b)=(1,-72) .
\end{array}
$$

prop:0 Proposition 6.6. The minimal order of an O-type Maya diagram $M=\mathrm{O}\left(\ell_{1}, \ell_{2}\right), \ell_{1}, \ell_{2} \in \mathbb{N}$, is $\max \left\{\ell_{1}, \ell_{2}\right\}$. If $\ell_{1} \leq \ell_{2}$, then the minimal-order pseudo-Wronskian is

$$
H_{M-3 \ell_{1}}= \pm\left|\begin{array}{cccc}
\tilde{H}_{2} & \tilde{H}_{3} & \ldots & \tilde{H}_{\ell_{2}+1} \\
\tilde{H}_{5} & \tilde{H}_{6} & \ldots & \tilde{H}_{\ell_{2}+4} \\
\vdots & \vdots & \ddots & \vdots \\
\tilde{H}_{3 \ell_{1}-1} & \tilde{H}_{3 \ell_{1}} & \ldots & \tilde{H}_{3 \ell_{1}+\ell_{2}-2} \\
H_{2} & H_{2}^{\prime} & \ldots & H_{2}^{\left(\ell_{2}-1\right)} \\
H_{5} & H_{5}^{\prime} & \ldots & H_{5}^{\left(\ell_{2}-1\right)} \\
\vdots & \vdots & \ddots & \vdots \\
H_{3 \ell_{2}-3 \ell_{1}-1} & H_{3 \ell_{2}-3 \ell_{1}-1}^{\prime} & \ldots & H_{3 \ell_{2}-3 \ell_{1}-1}^{\left(\ell_{2}-1\right)}
\end{array}\right|
$$

If $\ell_{1}>\ell_{2}$, then the minimal-order pseudo-Wronskian is

$$
H_{M-3 \ell_{2}}= \pm\left|\begin{array}{cccc}
\tilde{H}_{2} & \tilde{H}_{3} & \ldots & \tilde{H}_{\ell_{1}+1} \\
\tilde{H}_{5} & \tilde{H}_{6} & \ldots & \tilde{H}_{\ell_{1}+4} \\
\vdots & \vdots & \ddots & \vdots \\
\tilde{H}_{3 \ell_{2}-1} & \tilde{H}_{3 \ell_{2}} & \ldots & \tilde{H}_{3 \ell_{2}+\ell_{1}-2} \\
H_{1} & H_{1}^{\prime} & \ldots & H_{1}^{\left(\ell_{2}-1\right)} \\
H_{4} & H_{4}^{\prime} & \ldots & H_{4}^{\left(\ell_{2}-1\right)} \\
\vdots & \vdots & \ddots & \vdots \\
H_{3 \ell_{1}-3 \ell_{2}-2} & H_{3 \ell_{1}-3 \ell_{2}-2}^{\prime} & \ldots & H_{3 \ell_{1}-3 \ell_{2}-2}^{\left(\ell_{2}-1\right)}
\end{array}\right|
$$

Proof. Suppose that $\ell_{1} \leq \ell_{2}$, so that

$$
M_{+}=\left\{1,2,4,5, \ldots, 3 \ell_{1}-2,3 \ell_{1}-1\right\} \cup\left\{3 \ell_{1}+2,3 \ell_{1}+5, \ldots, 3 \ell_{2}-1\right\} .
$$



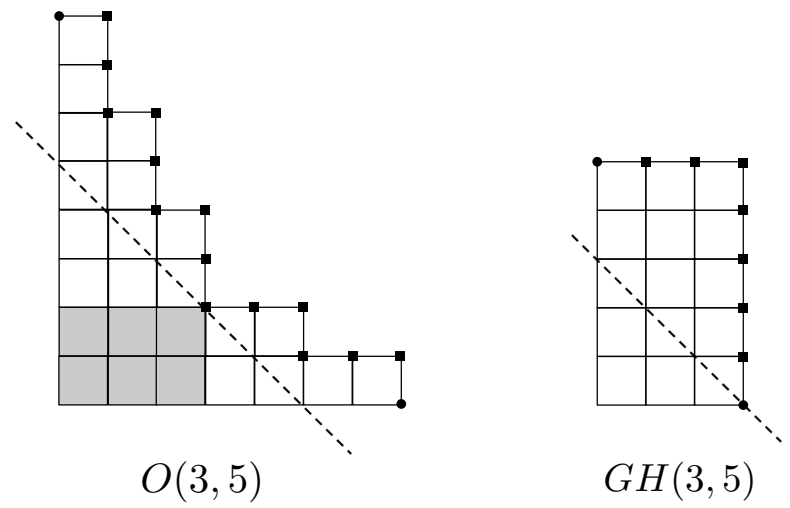

Figure 4. Partitions and minimal order pseudo-Wronskians for two generalized Hermite and Okamoto polynomials. Minimal order representations for these polynomials are given by Durfee symbols $[6,4,2 \mid 4,2]_{3 \times 2}$ for $O(3,5)$ and $[5,5,5 \mid \emptyset]_{3 \times 0}$ for $G H(3,5)$.

fig: okamoto

The inside corners are located at $0,3,6, \ldots, 3 \ell_{1}$ and then $3 \ell_{1}+3,3 \ell_{1}+6, \ldots, 3 \ell_{2}$. The corresponding girths initially decrease: $\ell_{1}+\ell_{2},+\ell_{2}-1, \ldots, \ell_{2}+1, \ell_{2}$ and increase by +1 thereafter.

Suppose that $\ell_{1}>\ell_{2}$, so that

$$
M_{+}=\left\{1,2,4,5, \ldots, 3 \ell_{2}-2,3 \ell_{2}-1\right\} \cup\left\{3 \ell_{2}+1,3 \ell_{2}+5, \ldots, 3 \ell_{1}-2\right\}
$$

The inside corners are $0,3,6, \ldots, 3 \ell_{2}$ and then $3 \ell_{2}+2,3 \ell_{2}+5, \ldots, 3 \ell_{1}-1$. The corresponding girths initially decrease: $\ell_{1}+\ell_{2}, \ell_{1}+\ell_{2}-1, \ldots, \ell_{1}+1, \ell_{1}$ and increase by +1 thereafter.

Figure 4 contains a graphical illustration for the minimal order pseudo-Wronskians described by Propositions 6.5 and 6.6 for the Generalized Hermite and Okamoto polynomials, respectively. We see thus that in the case of generalized Hermite polynomials, the minimal order occurs always for the partition itself or its conjugate partition, and therefore it is always a true Wronskian. In the example of the figure for $G H(3,5)$, the minimal order according to Proposition 6.5 must be $\min (3,5)=3$ :

$$
G H(3,5)=\mathrm{Wr}\left(H_{3}, H_{4}, H_{5}, H_{6}, H_{7}\right)=\frac{1}{18432} \mathrm{Wr}\left(\tilde{H}_{5}, \tilde{H}_{6}, \tilde{H}_{7}\right)
$$

On the contrary, for Okamoto polynomials, the minimal order is generically a pseudo-Wronskian. In the example of the figure, for $O(3,5)$ that has order 8 , the equivalent minimal order pseudoWronskian has order $\max (3,5)=5$ and corresponds to the Durfee symbol $[6,4,2 \mid 4,2]_{3 \times 2}$.

$$
O(3,5)=\operatorname{Wr}\left(H_{1}, H_{2}, H_{4}, H_{5}, H_{7}, H_{8}, H_{11}, H_{14}\right) \propto \mathrm{e}^{-3 x^{2}} \mathrm{Wr}\left(\mathrm{e}^{x^{2}} \tilde{H}_{8}, \mathrm{e}^{x^{2}} \tilde{H}_{5}, \mathrm{e}^{x^{2}} \tilde{H}_{2}, H_{2}, H_{5}\right)
$$


Example 6.2. For the rational solutions of $\mathrm{P}_{\mathrm{IV}}$ corresponding to the Okamoto class, take $\ell_{1}=$ $1, \ell_{2}=2$ and set

$$
\begin{aligned}
& M_{0}=\mathrm{O}(1,2)=\mathbb{Z}_{-} \cup\{1,2,5\} \\
& M_{1}=\mathrm{O}(0,1)=\mathbb{Z}_{-} \cup\{2\} \\
& M_{2}=\mathrm{O}(2,2)=\mathbb{Z}_{-} \cup\{1,2,4,5\} \\
& M_{3}=\mathrm{O}(1,3)=\mathbb{Z}_{-} \cup\{1,2,5,8\} .
\end{aligned}
$$

The corresponding Wronskian determinants have order $3,1,4,4$, respectively. The minimal order description is in term of pseudo-Wronskian determinants of order $2,1,2,3$, respectively. By a direct calculation,

$$
\begin{aligned}
H_{M_{0}-3} & =\left|\begin{array}{ll}
\tilde{H}_{2} & \tilde{H}_{2} \\
H_{2} & H_{2}^{\prime}
\end{array}\right|=-8 x\left(4 x^{4}-5\right) \\
H_{M_{1}} & =H_{2}=2\left(2 x^{2}-1\right) \\
H_{M_{2}-6} & =\left|\begin{array}{ll}
\tilde{H}_{5} & \tilde{H}_{6} \\
\tilde{H}_{2} & \tilde{H}_{3}
\end{array}\right|=-48\left(8 x^{6}+20 x^{4}+10 x^{2}+5\right) \\
H_{M_{3}-3} & =\left|\begin{array}{lll}
\tilde{H}_{2} & \tilde{H}_{3} & \tilde{H}_{4} \\
H_{2} & H_{2}^{\prime} & H_{2}^{\prime \prime} \\
H_{5} & H_{5}^{\prime} & H_{5}^{\prime \prime}
\end{array}\right|=192\left(32 x^{10}-80 x^{8}-80 x^{6}+200 x^{4}-150 x^{2}-25\right)
\end{aligned}
$$

Setting $x=t / \sqrt{3}$, the corresponding rational solutions (39) (40) are

$$
\begin{array}{ll}
y_{1}=-\frac{2 t}{3}+\frac{16 t^{3}}{4 t^{4}-45}+\frac{1}{t}-\frac{4 t}{2 t^{2}-3}, & (a, b)=\left(3,-\frac{32}{9}\right), \\
y_{2}=-\frac{2 t}{3}+\frac{16 t^{3}}{4 t^{4}-45}+\frac{1}{t}-\frac{12\left(4 t^{5}+20 t^{3}+15 t\right)}{8 t^{6}+60 t^{4}+90 t^{2}+135}, & (a, b)=\left(-1,-\frac{128}{9}\right), \\
y_{3}=-\frac{2 t}{3}+\frac{16 t^{3}}{4 t^{4}-45}+\frac{1}{t}-\frac{20\left(16 t^{9}-96 t^{7}-216 t^{5}+1080 t^{3}-1215 t\right)}{32 t^{10}-240 t^{8}-720 t^{6}+5400 t^{4}-12150 t^{2}-6075}, & (a, b)=\left(-5,-\frac{32}{9}\right) .
\end{array}
$$

\section{AcKnowledgements}

The research of DGU has been supported in part by Spanish MINECO-FEDER Grant MTM201565888-C4-3, the ICMAT-Severo Ochoa project SEV-2015-0554 and the BBVA Foundation Grant for researchers and cultural creators. The research of RM was supported in part by NSERC grant RGPIN-228057-2009. DGU and RM would like to thank Université de Lorraine for their hospitality during their visit in the summer of 2015 where many of the results in this paper where first obtained.

\section{REFERENCES}

[1] V. E. Adler, A modification of Crum's method, Theor. Math. Phys. 101 (1994), 1381-1386.

[2] V. E. Adler, Nonlinear chains and Painlevé equations, Physica D 73.4 (1994), 335-351.

[3] G. E. Andrews, Generalization of the Durfee square, J. London Math. Soc. 3 (1971), 563-570.

[4] G. E. Andrews and K. Eriksson Integer partitions, Cambridge University Press, 2004.

[5] G. E. Andrews, The theory of partitions, Cambridge University Press, New York, 1998.

[6] B. Bakalov, E. Horozov and M. Yakimov, Bispectral algebras of commuting ordinary differential operators. Commun. Math. Phys. 190(2) (1997) 331-373.

[7] W. Chu and L. Di Claudio, Classical partition identities and basic hypergeometric series, Edizioni del Grifo, 2004. 
[8] P.A. Clarkson, The fourth Painlevé equation and associated special polynomials. J. Math. Phys. 44 (2003), 5350-5374.

[9] P.A. Clarkson, Vortices and polynomials, Stud. Appl. Math. 123 (2009) 37-62.

[10] M. M. Crum, Associated Sturm-Liouville systems, Quart. J. Math. Oxford Ser. (2) 6 (1955), $121-127$.

[11] P. A. Deift, Applications of a commutation formula, Duke Math. J. 45(2) (1978), $267-310$.

[12] J. J. Duistermaat and F. A. Grübaum, Differential equations in the spectral parameter, Commun. Math. Phys. 103(2) (1986), 177-240.

[13] A. J. Durán, Higher order recurrence relation for exceptional Charlier, Meixner, Hermite and Laguerre orthogonal polynomials, Integr. Transf. Spec. F. 26 (2015), 357-376.

[14] G. Felder, A. D. Hemery, and A. P. Veselov, Zeros of Wronskians of Hermite polynomials and Young diagrams, Physica D 241 (2012), 2131-2137.

[15] G. V. Filipuk and P. A. Clarkson, The symmetric fourth Painlevé hierarchy and associated special polynomials, Stud. Appl. Math. 121(2) (2008), 157-188.

[16] W. Fulton and J. Harris, Representation theory. A first course. Graduate Texts in Mathematics 129. Readings in Mathematics. Springer-Verlag, New York, 1991.

[17] M. Feigin and D. Johnston, A class of Baker-Akhiezer arrangements, Commun. Math. Phys., 328(3) (2014), 1117-1157.

[18] N. C. Freeman, and J. J. C. Nimmo, Soliton solutions of the Korteweg de Vries and the Kadomtsev-Petviashvili equations: the Wronskian technique, Proc. Royal Soc. London A $\mathbf{3 8 9}$ (1983), 319-329.

[19] M. A. García-Ferrero, D. Gómez-Ullate, Oscillation theorems for the Wronskian of an arbitrary sequence of eigenfunctions of Schrödingers equation, Lett. Math. Phys. 105(4) (2015), 551-573.

[20] M. A. García-Ferrero, D. Gómez-Ullate, and R. Milson, A Bochner type classification theorem for exceptional orthogonal polynomials, (2016) arXiv:1603.04358 [math.CA].

[21] D. Gómez-Ullate, N. Kamran, and R. Milson, An extended class of orthogonal polynomials defined by a SturmLiouville problem, J. Math. Anal. Appl. 359 (2009), 352-367.

[22] D. Gómez-Ullate, N. Kamran, and R. Milson, An extension of Bochner's problem: Exceptional invariant subspaces, J. Approx. Theory 162 (2010), 987-1006.

[23] D. Gómez-Ullate, N. Kamran, and R. Milson, A conjecture on exceptional orthogonal polynomials, Found. Comput. Math. 13 (2012), 615-666.

[24] D. Gómez-Ullate, F. Marcellán, and R. Milson, Asymptotic and interlacing properties of zeros of exceptional Jacobi and Laguerre polynomials, J. Math. Anal. Appl. 399 (2013), 480-495.

[25] D. Gómez-Ullate, Y. Grandati, and R. Milson, Rational extensions of the quantum harmonic oscillator and exceptional Hermite polynomials, J. Phys. A: Math. Gen. 47 (2014), 015203.

[26] D. Gómez-Ullate, A. Kasman, A. B. J. Kuijlaars and R. Milson, Recurrence Relations for Exceptional Hermite Polynomials, J. Approx. Theory 204 (2016), 1-16.

[27] D. Gómez-Ullate, Y. Grandati, and R. Milson, Rational solutions of Painlevé IV and $V$ and Exceptional Orthogonal Polynomials, in preparation.

[28] D. Gómez-Ullate, Y. Grandati, and R. Milson, Shape invariance and equivalent Jacobi-Trudi representations for $X-O P$, in preparation.

[29] Y. Grandati, Exceptional orthogonal polynomials and generalized Schur polynomials, J. Math. Phys. 55(8) (2014), 083509.

[30] F. A. Grübaum and L. Haine, Some functions that generalize the AskeyWilson polynomials, Commun. Math. Phys. 184(1) (1997) 173-202.

[31] A. D. Hemery and A. P. Veselov, Whittaker-Hill equation and semifinite-gap Schrödinger operators, J. Math. Phys. 51 (2010), 072108.

[32] C.-L. Ho, Dirac(-Pauli), Fokker-Planck equations and exceptional Laguerre polynomials, Ann. Phys. 326 (2011), 797-807.

[33] Á. P. Horváth, The electrostatic properties of zeros of exceptional Laguerre and Jacobi polynomials and stable interpolation, J. Approx. Theory 194 (2015), 87-107.

[34] M. V. Demina and N. A. Kudryashov, Multiparticle dynamical systems and polynomials, Reg. Chaotic Dyn. 21 (2016), 351-366.

[35] A. B. J. Kuijlaars and R. Milson, Zeros of exceptional Hermite polynomials, J. Approx. Theory 200 (2015), $28-39$.

[36] D. Gómez-Ullate, Y. Grandati, and R. Milson, Extended Krein-Adler theorem for the translationally shape invariant potentials, J. Math. Phys. 55 (2014), 043510. 
[37] S. Karlin and G. Szegő, On certain determinants whose elements are orthogonal polynomials, J. Analyse Math. 8 (1960), 1-157.

[38] I. Marquette and C. Quesne, New families of superintegrable systems from Hermite and Laguerre exceptional orthogonal polynomials, J. Math. Phys. 54 (2013), 042102.

[39] V. B. Matveev and M. A. Salle, Darboux transformations and solitons. Berlin: Springer-Verlag, 1991.

[40] H. Miki and S. Tsujimoto, A new recurrence formula for generic exceptional orthogonal polynomials, J. Math. Phys. 56 (2015), 033502.

[41] M. Noumi, Painlev equations through symmetry. AMS-Springer Mathematical Monographs 223, 2004.

[42] A.A. Oblomkov, Monodromy-free Schrödinger operators with quadratically increasing potentials, Theor. Math. Phys., 121(3):1574-1584, 1999.

[43] S. Odake, Equivalences of the multi-indexed orthogonal polynomials, J. Math. Phys. 55 (2014), 013502.

[44] J. B. Olsson, Combinatorics and representations of finite groups. Vol. 20 Fachbereich Mathematik, Universität Essen, 1993.

[45] S. Odake and R. Sasaki, Infinitely many shape invariant potentials and new orthogonal polynomials, Phys. Lett. B 679 (2009), 414-417.

[46] S. Odake, Recurrence Relations of the Multi-Indexed Orthogonal Polynomials : III, J J. Math. Phys. 57 (2016), 023514 .

[47] Y. Ohta, J. Satsuma, D. Takahashi and T. Tokihiro, An elementary introduction to Sato theory, Progress of theoretical physics supplement, 94 (1988) 210-241.

[48] S. Post, S. Tsujimoto, and L. Vinet, Families of superintegrable Hamiltonians constructed from exceptional polynomials, J. Phys. A: Math. Gen. 45 (2012), 405202.

[49] C. Quesne, Solvable rational potentials and exceptional orthogonal polynomials in supersymmetric quantum mechanics, SIGMA 5 (2009).

[50] A. Schulze-Halberg and B. Roy, Darboux partners of pseudoscalar Dirac potentials associated with exceptional orthogonal polynomials, Ann. Phys. 349 (2014), 159-170.

[51] A. N. Sergeev, and A. P. Veselov, Jacobi-Trudy formula for generalized Schur polynomials, Mosc. Math. J. 14(1) (2014), 161-168.

[52] G. Szegő, Orthogonal Polynomials, Colloquium Publications, vol. 23, American Mathematical Society, 1939.

[53] K. Takemura, Multi-indexed Jacobi polynomials and Maya diagrams, J. Math. Phys. 55 (2014), 113501.

[54] A. P. Veselov and A. B. Shabat, Dressing chains and spectral theory of the Schrodinger operator, Funktsional. Anal. i Prilozhen. 27(2) (1993), 1-21.

[55] J. P. Zubelli and F. Magri, Differential equations in the spectral parameter, Darboux transformations and a hierarchy of master symmetries for KdV. Commun. Math. Phys. 141(2) (1991), 329-351.

Departamento de Física Teórica II, Universidad Complutense de Madrid, 28040 Madrid, Spain. SPAIN.

Instituto de Ciencias Matemáticas (CSiC-UAM-UC3M-UCM), C/ Nicolas Cabrera 15, 28049 Madrid,

LCP A2MC, Université de Lorraine, 1 Bd Arago, 57078 Metz, Cedex 3, France.

Department of Mathematics and Statistics, Dalhousie University, Halifax, NS, B3H 3J5, Canada.

E-mail address: david.gomez-ullate@icmat.es, grandati@univ-metz.fr, rmilson@dal.ca 